\title{
ALGORITMO MATEMÁtICO DE LA SEPARACIÓN DE UN SISTEMA BINARIO MEDIANTE ELECTRODIÁLISIS
}

\author{
MAthematical algorithm For the SEPARATION OF A BINARY SYSTEM BY \\ ELECTRODIALYSIS
}

Yovanny Morales Hernández*

Jaime Arturo Calvache**

Jhoan Cifuentes Aguilera***

Neyfe Murcia Berdugo****

Recibido: 11 de enero de 2017

Aceptado: 25 de abril de 2017

\section{Resumen}

La electrodiálisis es una técnica de separación usada para la recuperación de sales, ácidos y bases de mezclas acuosas. En este trabajo se busca implementar un modelo matemático que, usando modelos teóricos y diferentes simplificaciones, represente una separación mediante electrodiálisis de una disolución acuosa binaria. Las ecuaciones planteadas modelan los fenómenos de transferencia de masa que ocurren a lo largo del módulo de electrodiálisis y tienen en cuenta algunas hipótesis simplificativas para facilitar el modelamiento del proceso. El modelo también usa ecuaciones que describen el consumo energético de la separación, el cual, junto a la pureza obtenida para el soluto, permite estimar la viabilidad de la técnica.

Palabras clave: celda de electrodiálisis, membranas de intercambio iónico, modelo matemático.

\section{Abstract}

Electrodialysis is a used separation technique for water desalination and recovery of salts, acids and aqueous mixtures bases. This paper aims to implement a mathematical model using theoretical models and simplifications, can represent a separation by electrodialysis for a binary aqueous solution. Equations raised model phenomena mass transfer occurring along the electrodialysis

\footnotetext{
* Ingeniero químico. Docente investigador, grupo de investigación en Procesos de Separación no Convencionales (GPS), Fundación Universidad de América. yovanny.morales@profesores.umaerica.edu.co

${ }^{* *}$ Ingeniero químico, magíster en Ingeniería Química. Docente investigador, grupo de investigación en Procesos de Separación no Convencionales (GPS), Fundación Universidad de América.

jaime.arturo@profesores.umaerica.edu.co

*** Ingeniero químico. Coinvestigador, grupo de investigación en Procesos de Separación no Convencionales (GPS), Fundación Universidad de América. jhoan.cifuentes@estudiantes.umaerica.edu.co

${ }_{* * * *}$ Ingeniera química. Coinvestigador, grupo de investigación en Procesos de Separación no Convencionales (GPS), Fundación Universidad de América.neyfe.murcia@estudiantes.umaerica.edu.co
} 
module considering some simplifying assumptions in order to facilitate the modeling process. The model also contains equations describing energy consumption of the separation, which, along with the purity obtained for the solute, allows to estimate the viability of the technique.

Keywords: electrodialysis cell, ion exchange membranes, mathematical model.

\section{INTRODUCCIÓN}

La electrodiálisis es una técnica de separación electroquímica basada en dos principios: la aplicación de un campo eléctrico continuo y la transferencia de iones a través de membranas de intercambio iónico.

El gradiente o la fuerza impulsora utilizada es el potencial eléctrico, pues al aplicarse permite la migración iónica a través de las membranas concentrando los iones de interés en un compartimiento existente entre las membranas al que se le denomina concentrado, mientras que la solución alimentada entra por el compartimiento denominado diluido. (Medina, 2007, p. 23)

Posteriormente, se lleva toda la solución obtenida de los compartimientos de concentrado a un tanque de concentrado aislado de las membranas de intercambio iónico.

Un módulo de electrodiálisis consta principalmente de "un par de electrodos encargados de conducir la corriente eléctrica, membranas de intercambio catiónico (permeables a cationes) y membranas de intercambio aniónico (permeables a aniones) ubicados de manera alternada, los compartimientos de diluido y de concentrado" (Bonet, 2010, p. 17). Para modelar matemáticamente el proceso "es necesario establecer un volumen de control y realizar algunas simplificaciones sugeridas por modelos como el de la termodinámica irreversible o el de Nernst-Planck" (Hábová, Melzoch, Rychtera y Sekavová, 2004, p. 363). Una vez se establecen las simplificaciones, se desarrollan las relaciones de transferencia de masa que están limitadas por los fenómenos de migración iónica; se realizan los balances de materia y especies, y se definen las relaciones de consumo energético para el sistema electrolítico. Después de definir el modelo matemático para el sistema electrólitico, se establece un algoritmo para su solución numérica.

\section{Métodos}

\section{Simplificaciones}

Con el fin de facilitar el modelamiento matemático de una separación mediante electrodiálisis, se tomaron en cuenta las siguientes simplificaciones:

- El caudal de la alimentación y de la corriente de concentrado se debe mantener constante a lo largo del proceso, así como su temperatura.

- Las pérdidas energéticas hacia el exterior de la pila de electrodiálisis se menosprecian.

- Se asume que no hay pérdidas de fluido a lo largo de todo el módulo.

- Solo hay dos especies en disolución: anión y catión del soluto en disolución acuosa.

- El valor de la intensidad de la corriente siempre será menor al valor de la intensidad de la corriente límite.

- Los compartimientos de concentrado y diluido, así como el tanque de concentrado, son considerados como espacios de mezcla perfecta, lo que conlleva que la concentración en ellos sea uniforme y que la concentración de la salida sea la misma concentración que existe internamente en el tanque y los compartimientos.

- Se ignora el efecto de los compartimientos de electrodo, debido a que es despreciable con respecto al elevado número de pares de celdas que suelen utilizarse en una pila de electrodiálisis. 
- Las superficies de las membranas de intercambio iónico se consideran planas y con una sección transversal uniforme.

- Las membranas de intercambio iónico son paralelas entre sí.

- El funcionamiento de las membranas de intercambio iónico es ideal, debido a que las membranas de intercambio aniónico solo permiten el paso a los aniones y las de intercambio catiónico solo son permeables a los cationes.

- No se tienen en cuenta los efectos asociados a los fenómenos del fouling y scaling.

- Los coeficientes de difusión permanecen constantes a lo largo del modelamiento.

- Todos los compartimientos se consideran idénticos, por lo que el coeficiente de transferencia de masa será igual para todos.

- Se sigue el modelo Nernst-Planck,

[...] el cuál no es más que una simplificación de la termodinámica irreversible. Propone despreciar las interacciones entre los iones, así como el transporte por convección causado por el gradiente de presión entre los compartimientos. También sugiere utilizar coeficientes de actividad con valor de 1. (Bonet, 2010, p. 43)

\section{Fenómenos de transferencia de masa}

Una de las razones por las que el modelamiento del proceso aumenta su complejidad es que se presentan varios fenómenos de transferencia de masa simultáneamente, por lo que es necesario comprender a qué se refiere cada uno de ellos, y así deducir ecuaciones que representen su efecto en el sistema.

El fenómeno de la migración iónica hace referencia a la "cantidad de iones que se transfieren de un compartimiento a otro a través de una membrana como consecuencia de la aplicación de una fuerza impulsora, que en el caso de la electrodiálisis es el gradiente de potencial eléctrico" (Matsuura, 1993). Se debe tener en cuenta que los iones no son las únicas especies existentes en la mezcla, pues se encuentran presentes en una disolución acuosa, por lo que arrastrarán junto con ellos moléculas de solvente, en este caso agua; a este tipo de transporte se le denomina electroósmosis. Ambos mecanismos de transferencia se pueden representar partiendo de la ley de Faraday.

Al aplicar la ley de Faraday en la electrodiálisis:

[...] se está enunciando que un mol del compuesto que se encuentra diluido necesita que pase por el stack una corriente eléctrica de 96485.33 A por segundo para migrar del compartimiento de diluido al compartimiento de concentrado, esto si se asume un rendimiento eléctrico de $100 \%$. (Bonet, 2010, p. 25)

En las ecuaciones 1,2,3 y 4 se representan los fenómenos causados por el gradiente de potencial eléctrico, donde j es el agua, i es el compuesto diluido, , es el catión y_ es el anión.

$$
\begin{gathered}
M=\frac{\eta^{*} I}{z j * F} *(3600 \text { segundos })=M_{+}+M_{-} \\
M_{+}=t_{+} * \frac{\eta^{*} I}{z j^{*} F} *(3600 \text { segundos }) \\
M_{-}=t_{-} * \frac{\eta^{*} I}{z j^{*} F} *(3600 \text { segundos }) \\
t_{+}+t_{-}=1
\end{gathered}
$$


La ecuación 1 representa la migración iónica total $M$ en función de la ecuación de Faraday, donde $\eta$ es el rendimiento eléctrico, $I$ es la intensidad de corriente (A), zj es la carga iónica de $j$, $F$ es la constante de Faraday $(\mathrm{C} / \mathrm{mol}) M_{+}$y $M_{-}$y representan la migración iónica del catión y el anión, respectivamente. Las ecuaciones 2 y 3 modelan la migración iónica de cada ion teniendo en cuenta el término $t$, que es el número de transporte e "indica la fracción de corriente transportada por los cationes o por los aniones, además debe mencionarse que su valor se ve afectado en menor proporción por la temperatura o por la concentración" (Mayorga y Ariza, 2013, p. 50). Teniendo en cuenta las ecuaciones 1, 2 y 3 es posible deducir que al sumar los números de transporte de aniones y cationes se obtendrá un valor de 1.

Para determinar el número de transporte de cada ion es necesario conocer la conductividad equivalente $\lambda$, tanto del anión como del catión, y utilizar las ecuaciones 6 y 7 para obtener un valor específico; mientras que para determinar el número de transporte del agua se deben conocer los números de hidratación primario $h$ de los iones, o bien, buscar su valor en referentes bibliográficos con el fin de utilizarlos en la ecuación 8. La electroósmosis también se modela partiendo de la ley de Faraday; igualmente, los parámetros necesarios son muy similares a los utilizados en los fenómenos de migración iónica (la ecuación 5 representa este efecto).

$$
\begin{gathered}
M i=t_{i} * \frac{\eta^{*} I}{z j^{*} F} *(3600 \text { segundos }) \\
t_{+}=\frac{\lambda_{+}}{\lambda_{+}+\lambda_{-}} \\
t_{-}=\frac{\lambda_{-}}{\lambda_{+}+\lambda_{-}} \\
t_{i}=h_{+}+b_{-}
\end{gathered}
$$

Los fenómenos mencionados y la manera como se encuentran dispuestas las membranas provocan que algunos compartimientos se vayan concentrando y otros diluyendo, por lo que se generará un gradiente de concentración, este gradiente conduce a que se presente un flujo de iones desde el compartimiento más concentrado al compartimiento más diluido, flujo que se denomina difusión iónica.

Las moléculas de agua sufren un efecto similar, pues tienden a transportarse de la zona más diluida a la concentrada. Esto se conoce como ósmosis. Los anteriores mecanismos, al estar ligados con fenómenos de difusividad, se pueden modelar a partir de la ley de Fick.

La ley de Fick representará el flujo de iones y de agua a través de las membranas debido a un gradiente de concentración. Las ecuaciones 9,10,11 y 12 representan la difusión de cada ion y del agua.

$$
\begin{gathered}
D=D_{+}+D_{-} \\
D_{+}=D_{++m i c} * A * \frac{d C_{m i c}}{d X_{m i c}}=\frac{D_{+} m i c}{\sigma_{m i c}} * S
\end{gathered}
$$




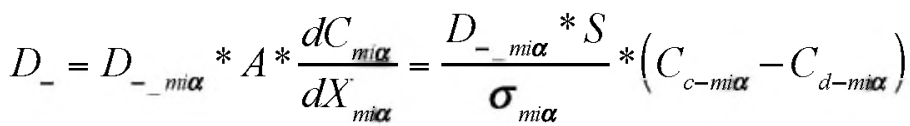

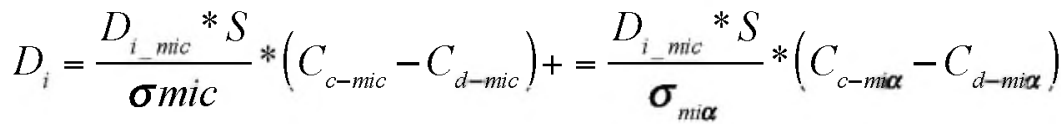

Para modelar la difusión se necesita de un:

[...] coeficiente de difusividad de la especie que se estudia en el medio que se busca difundir, un área efectiva por donde se llevará a cabo el fenómeno, y un gradiente de concentración que se expresa como el cociente entre una diferencia de concentraciones y una diferencia de distancias. (Bonet, 2010, p. 27)

Al acoplar todos los parámetros se obtienen las ecuaciones 10 y 11, mientras que la ecuación 9 no es más que la suma de la difusión iónica del anión y el catión. Para la difusión de los iones, $D_{+}$y $D_{-}\left(\mathrm{mol}_{10 n} / \mathrm{h}\right)$, es necesario el coeficiente de difusión del ion a través de la membrana que es selectiva a este, $D_{+_{-} \text {mic }}$ y $D_{- \text {mia }}\left(\mathrm{dm}^{2} / \mathrm{s}\right)$, la superficie efectiva de la membrana, $S\left(\mathrm{dm}^{2}\right)$, la diferencia de concentraciones en cada extremo de la membrana, $\ddot{d}_{C \text { mia }}$ y $\dot{d}_{C m i c}(\mathrm{~mol} \mathrm{j} / \mathrm{L})$, y el espesor de la membrana selectiva, $\sigma_{m i c} \mathrm{y} \sigma_{\text {mia }}(\mathrm{dm})$. La ósmosis o difusión del agua se dará bajo el mismo principio; pero teniendo en cuenta que esta se difunde en ambas membranas, como se muestra en la ecuación 12. En la figura 1 se observa cómo se presentan los fenómenos de transferencia de masa en una pila de electrodiálisis donde la solución a tratar es salmuera.

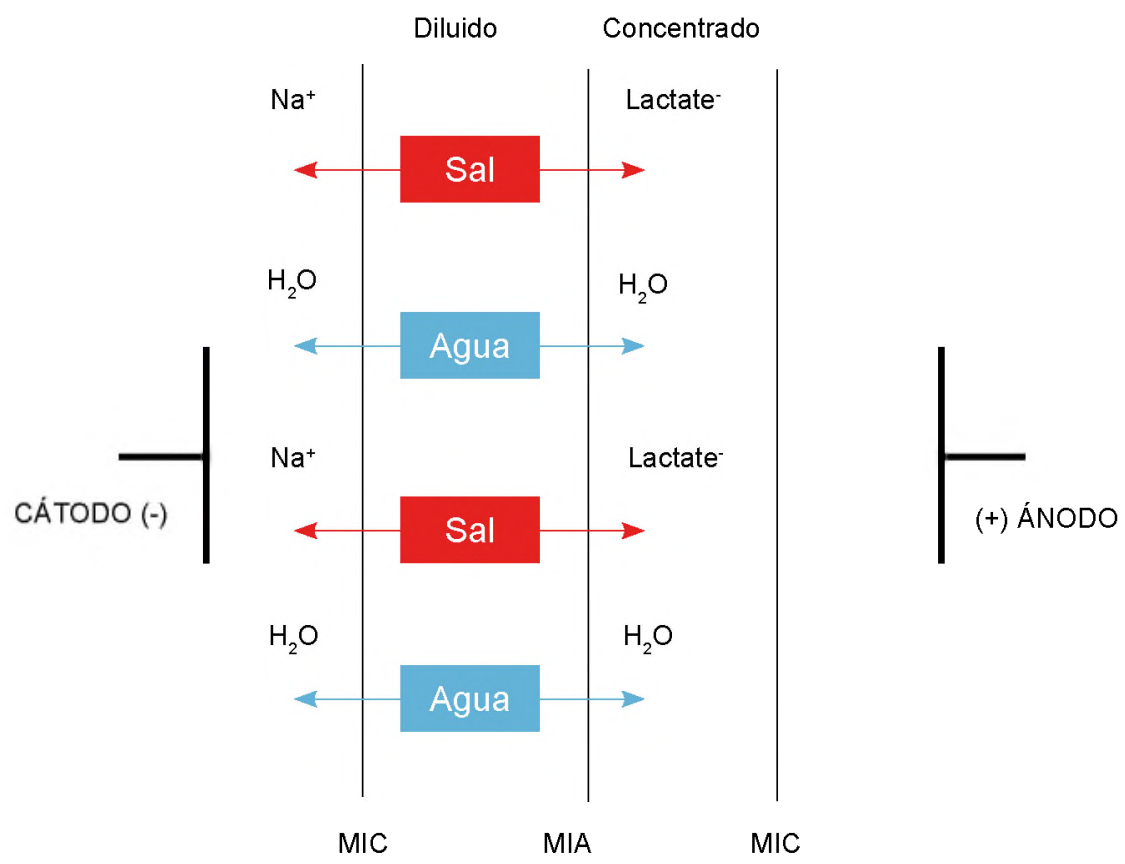

Figura 1. Transferencia de masa en un par de celdas de ED.

Fuente: tomado de Bonet (2010). 


\section{Balances de materia}

Para realizar los balances de materia es necesario establecer un volumen de control. En el caso de la electrodiálisis, el volumen de control (figura 2) corresponde un par de celdas (compartimiento de diluido y de concentrado, membrana de intercambio catiónica y aniónica) y el tanque de concentrado.

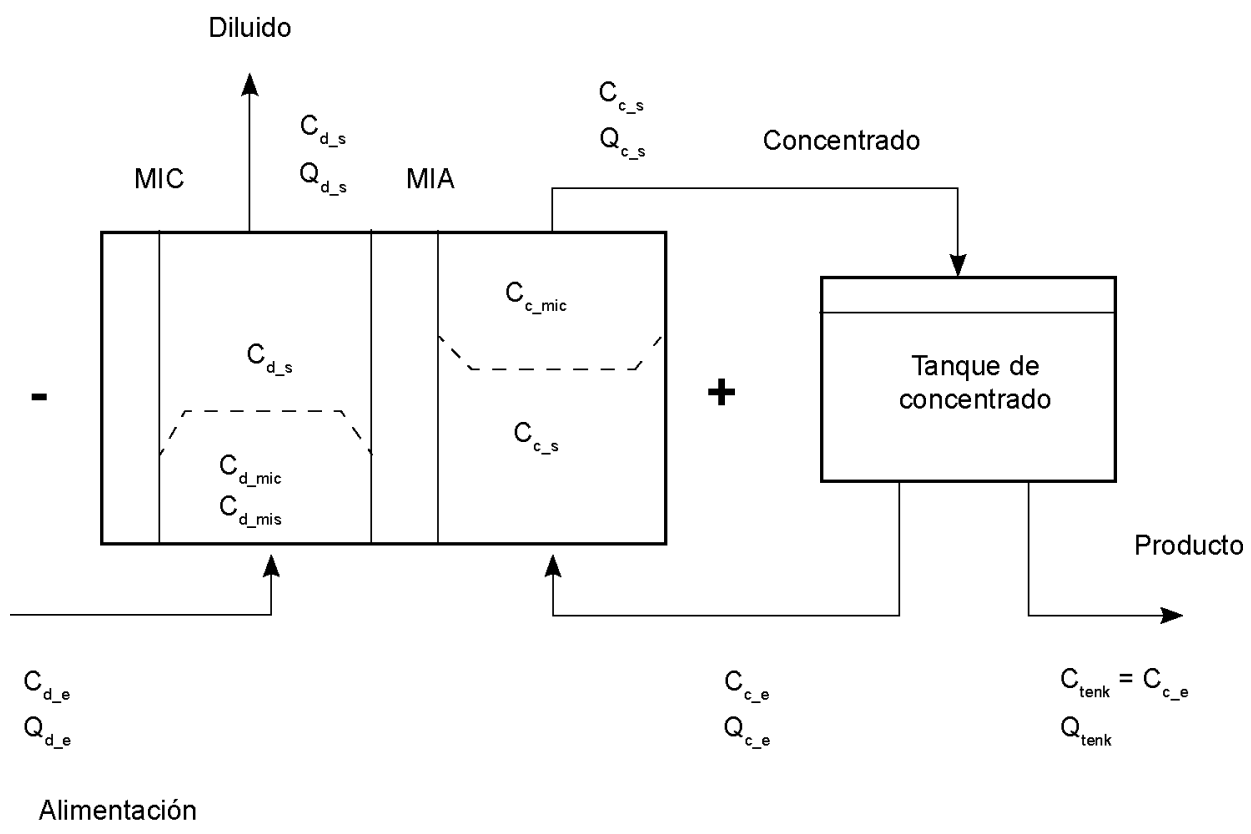

Figura 2. Volumen de control.

Fuente: tomado de Bonet (2010).

Se realizan balances de materia para cada sección del par de celdas, es decir, balance global y balance para el soluto en el compartimiento de concentrado, de diluido y en el tanque de concentrado. Adicionalmente, se deben tener en cuenta los fenómenos de transferencia de masa a través de las membranas.

Para los balances en el compartimiento de concentrado se iniciará con el balance global, como se representa en la ecuación 13.

$$
\begin{gathered}
\text { Salida-entrada }+ \text { Acumulación }=\text { Generación } \rightarrow \text { Salida }=\text { entrada } \\
Q_{c_{c s} s}=Q_{c_{-} e}+Q_{i} \\
Q_{i}=\left(D_{i}+M_{i}\right) *\left(\frac{P m_{i}}{\rho_{i}}\right)
\end{gathered}
$$

En la ecuación 14 se observa que los términos asociados al caudal de entrada y de salida para el compartimiento de concentrado son $Q_{c-e}$ y $Q_{c_{-S}}$, respectivamente; mientras que $Q_{i}$ representa el caudal de agua que ingresa a esa sección de la pila como consecuencia de los fenómenos de transferencia de masa por ósmosis y electroósmosis. Los términos $P m_{i}$ y $\rho_{i}$ se refieren al peso molecular 
del agua y a su densidad, y se utilizan con el objetivo de lograr un equilibrio dimensional en la ecuación 15.

Siguiendo una metodología similar se pueden inferir los balances globales para el compartimiento de diluido y el tanque de concentrado, así como se observa en las ecuaciones 16,17, 18 y 19.

$$
\begin{gathered}
Q_{d_{-} s}+Q_{i}=Q_{d_{-} e} \\
Q_{d_{-} s}+\left(D_{i}+M_{i}\right) *\left(\frac{P m_{i}}{\rho_{i}}\right)=Q_{d_{-} e} \\
Q_{T a n k}+Q_{c_{-} e}=Q_{c_{-} s} \\
Q_{T \text { ank }}+Q_{e_{-} e}=Q_{\tau_{-} e}+Q_{i} \rightarrow Q_{T \text { ank }}=Q_{i}
\end{gathered}
$$

En el balance del compartimiento de diluido (ecuación 16) se usa la relación expresada en la ecuación 15 con el fin de mostrar todos los fenómenos implicados, por lo que se obtiene la ecuación 17. La ecuación 19 tiene en cuenta la equivalencia presentada en la ecuación 14, con el objetivo de simplificar la expresión y hallar una relación mucho más sencilla a la presentada en la ecuación 18 entre el caudal del tanque $Q_{\text {Tank }}$ y los fenómenos asociados a la transferencia de moléculas de agua.

La ecuación 20 permite ver que en los balances de materia del soluto se tiene en cuenta el término de la acumulación para las tres secciones, lo que implica que haya un término diferencial en la ecuación. Asimismo, se deben tener en cuenta los fenómenos de transferencia de masa asociados a los iones, es decir, la migración y la difusión. En estos casos se deben tener en cuenta las concentraciones de cada caudal debido a que se trata de un balance de una especie específica.

$$
\text { Salida }+ \text { Acumulación }=\text { entrada }
$$

$$
\begin{aligned}
& \left(Q_{c_{-} s} * C_{s_{-} s}+D\right)+\left(N * V * \frac{d C_{c_{-} s}(t)}{d t}\right)=\left(Q_{c_{-} e} * C_{c_{-} e}+M\right) \\
& \left(Q_{d_{-} s} * C_{d_{-} s}+M\right)+\left(N * V * \frac{d C_{d_{-} s}(t)}{d t}\right)=\left(Q_{d_{-} e} * C_{d_{-} e}+D\right) \\
& \left(\left(Q_{c_{-} e} * Q_{T \text { ank }}\right) * C_{c_{-} e}\right)+\left(V_{T \text { ank }} * \frac{d C_{c_{-} e}(t)}{d t}\right)=\left(Q_{s_{-} s} * C_{c_{-} s}\right)
\end{aligned}
$$

Para que haya consistencia de unidades, el término diferencial se multiplica por el volumen de cada compartimiento y el número total de par de celdas $N$; en el caso del tanque solo se multiplica por su volumen $V_{T a n k}$. Una vez desarrollados los balances de materia del concentrado 21, del diluido 22 y del tanque 23 , se procede a determinar las concentraciones en la superficie de las membranas con el objetivo de poder dar solución a los fenómenos de difusividad iónica y ósmosis. 


\section{Concentraciones en la superficie de la membrana}

Si se supone que las membranas son $100 \%$ permeables a sus coiones, para hallar la concentración en las superficies de las membranas es posible utilizar las correlaciones 24, 25, 26 y 27 (Ortiz et ál., 2005, p. 70).

$$
\begin{aligned}
& C_{d_{-} m i a}=C_{d_{-} s}-\frac{\left(1-t_{-}\right) * \eta^{*} I}{z j * F^{*} k_{m}^{*} S} \\
& C_{c_{-} \text {mia }}=C_{s_{-} s}+\frac{\left(1-t_{-}\right) * \eta^{*} I}{z j * F^{*} k_{m}^{*} S} \\
& C_{d_{-} m i c}=C_{d_{-} s}-\frac{\left(1-t_{-}\right) * \eta \eta^{*} I}{z j * F^{*} k_{m} * S} \\
& C_{d_{-} m i c}=C_{d_{-} s}-\frac{\left(1-t_{-}\right) * \eta * I}{z j * F * k_{m}^{*} S}
\end{aligned}
$$

En las anteriores expresiones se puede observar la existencia de un nuevo término, $k_{m}$, que significa coeficiente de transferencia de masa y, como se mencionó en las simplificaciones, será constante a lo largo del módulo.

\section{Consumo energético del proceso}

Para estimar el consumo energético del proceso es necesario conocer diferentes variables asociadas tanto a los parámetros de entrada, como a las características de cada una de las membranas. Se debe considerar que hay resistencias eléctricas opuestas por las membranas y la misma disolución, como se representa en las ecuaciones 30 y 31, por lo que, al evaluar la resistencia total, todas se deben tener en cuenta, así como lo expresa la ecuación 29. Lo anterior permitirá obtener el valor del voltaje de toda la pila de electrodiálisis de acuerdo a la ecuación 28.

$$
\begin{gathered}
V=N^{*} I^{*} R \\
R=R_{d}+R_{c}+\frac{R_{\text {mia }}}{2 N^{*} S}+\frac{R_{\text {mic }}}{2 N^{*} S} \\
R_{d}=10 * \frac{L}{k_{d}^{*} S} \\
R_{c}=10 * \frac{L}{k_{c}^{*} S}
\end{gathered}
$$


Al respecto, Thang, Koschuh, Kulbe y Novalin (2005) afirman: "Las resistencias asociadas a los compartimientos de diluido y concentrado son $R_{d}$ y $R_{c}$, respectivamente y dependen de las conductividades de la disolución en cada compartimiento, adicionalmente también se ven influenciadas por la longitud del compartimiento $L "$ (p. 180). De manera que la suma de todos los términos relacionados a las resistencias es la resistencia total $R$.

El consumo energético representado por la ecuación 32 se define como la potencia consumida sobre la cantidad de producto obtenido en toneladas. Para determinar su valor se usan datos en estado estacionario (tiempo en el cual la disolución presente en el tanque de concentrado alcanza su máxima concentración) como medida de precaución debido a que el proceso comienza en el estado transitorio.

$$
E j=\frac{V * I}{C_{T \text { ank }} * Q_{T \text { ank }} * \frac{P m_{j}}{1000}}=\frac{V^{* I}}{C_{c_{-} e} * Q_{T \text { ank }} * \frac{P m_{j}}{1000}}
$$

Como se dijo en el párrafo anterior, los valores de la concentración del tanque $C_{\text {Tank }}$, su caudal $Q_{\text {Tank }}$ y el voltaje del proceso $V$ deben tomarse en el tiempo de estado estacionario.

\section{RESULTADOS Y DISCUSIÓN}

\section{Análisis de grados de libertad}

Se hace un análisis de grados de libertad con el objetivo de determinar cuántas y cuáles variables se deben especificar. Para dicho análisis se deben tener en cuenta los parámetros, las variables y las ecuaciones independientes (ver tabla 1).

Tabla 1. Análisis de grados de libertad para la separación mediante electrodiálisis de una disolución binaria

\begin{tabular}{ccccc}
\hline $\begin{array}{c}\text { Variables } \\
\text { desconocidas }\end{array}$ & $\begin{array}{c}\text { Ecuaciones (independientes + } \\
\text { relaciones) }\end{array}$ & Parámetros & $\begin{array}{c}\text { Grados de } \\
\text { libertad }\end{array}$ & Variables a especificar \\
\hline 31 & 24 & 28 & 7 & 7 \\
\hline
\end{tabular}

Del análisis es posible concluir que se deben especificar 7 variables con el objetivo de lograr que el problema tenga una solución única para determinadas condiciones. Las variables que se van a especificar son aquellas que en una aplicación real serían conocidas o serían parámetros de diseño. En este caso, las variables seleccionadas para ser especificadas son:

Intensidad de corriente, $I$.

Flujo de entrada del compartimiento de diluido, $Q_{d e}$.

Flujo de entrada del compartimiento de concentrado, $Q$.

Concentración a la entrada del compartimiento de diluido, $Q_{d}$.

Concentración a la entrada del compartimiento de concentrado, $Q_{c, e}$.

Volumen del tanque de concentrado, $V_{\text {Tank }}$.

Tiempo de ejecución del proceso, $t_{f}$

Una vez determinadas las variables que deben ser especificadas para poder desarrollar el modelo matemático, se debe proponer un orden de ejecución para cada ecuación o conjunto de ecuaciones. 


\section{Algoritmo de solución}

El algoritmo de solución propuesto indica que el primer paso es especificar los valores de las variables de entrada y conocer todos los parámetros que se necesitan en el modelo. Las primeras ecuaciones que se solucionarán son las que representan los fenómenos de migración iónica y electroósmosis, pues se mantendrán constantes a lo largo del desarrollo matemático debido a la naturaleza de las ecuaciones que las representan y a que todos los parámetros implicados en las ecuaciones 1 a 8 se consideran constantes.

Una vez hallado el valor para las variables mencionadas, se procederá a ejecutar el modelo desde un tiempo cero hasta un tiempo de ejecución $t_{f}$ Además de conocer los valores de todas las variables de entrada y parámetros, se deben acoplar o resolver simultáneamente las ecuaciones correspondientes a un sistema de 13 ecuaciones, donde 10 son lineales y 3 son diferenciales. Para resolver este sistema se recomienda discretizar las ecuaciones diferenciales y convertirlas en no lineales, con el fin de hacer más sencilla la metodología de cálculo. Se realiza un ciclo hasta que se cumpla la condición de que el tiempo sea igual al tiempo de ejecución del proceso.

Cuando se ha llegado al tiempo de ejecución $t$, se procede a realizar los cálculos del voltaje del módulo de electrodiálisis, pues hay variables desconocidas correspondientes a esta etapa del modelo que son necesarias para continuar con el algoritmo de solución. Finalmente, se determina el consumo energético del proceso usando los datos de estado estacionario (debido a que el modulo funcionará estacionariamente hasta el tiempo final de ejecución), que, junto a la concentración final obtenida en el tanque de concentrado, serán criterios para determinar la viabilidad o no de la separación mediante electrodiálisis

En la figura 3 se muestra un diagrama que representa el orden de ejecución del modelo matemático propuesto, mencionando los parámetros necesarios para dar solución a las ecuaciones correspondientes a cada una de las variables desconocidas y las condiciones que se deben alcanzar para finalizar un ciclo.

\section{Conclusiones}

Se establecieron varias simplificaciones en el modelo matemático propuesto con el objetivo de facilitar el modelamiento matemático y estimar el consumo energético y el valor de las concentraciones en cada etapa del proceso en diferentes intervalos de tiempo (entre $t=0$ y $t=t$ ) para disoluciones acuosas con un único soluto. Las simplificaciones están basadas principalmente en los modelos de la termodinámica irreversible y de Nerst-Planck, lo que implica que no hay interacciones entre los iones presentes en la solución, así como se desprecian las pérdidas de fluido a lo largo de todo el módulo de electrodiálisis y se consideran constantes parámetros como caudales, coeficientes de transferencia de masa y coeficientes de actividad. Al tener en cuenta todas las simplificaciones se logra modelar y solucionar de una forma más corta y sencilla el proceso de electrodiálisis.

El modelo está compuesto por balances de materia en los que se incluyen todos los fenómenos de transferencia de masa implicados en una separación mediante electrodiálisis (se desprecia el transporte por convección), así como las correlaciones que representan el consumo energético del proceso. Debido a la complejidad del modelo, se sugiere discretizar las ecuaciones diferenciales correspondientes a los balances de materia del soluto para solucionar el sistema de 13 ecuaciones de una forma más sencilla. El algoritmo presentado en la figura 3 representa la secuencia lógica de solución y las condiciones que se deben cumplir para finalizar determinados ciclos. El modelo matemático propuesto puede representar el proceso de separación mediante electrodiálisis en distintas mezclas binarias. 


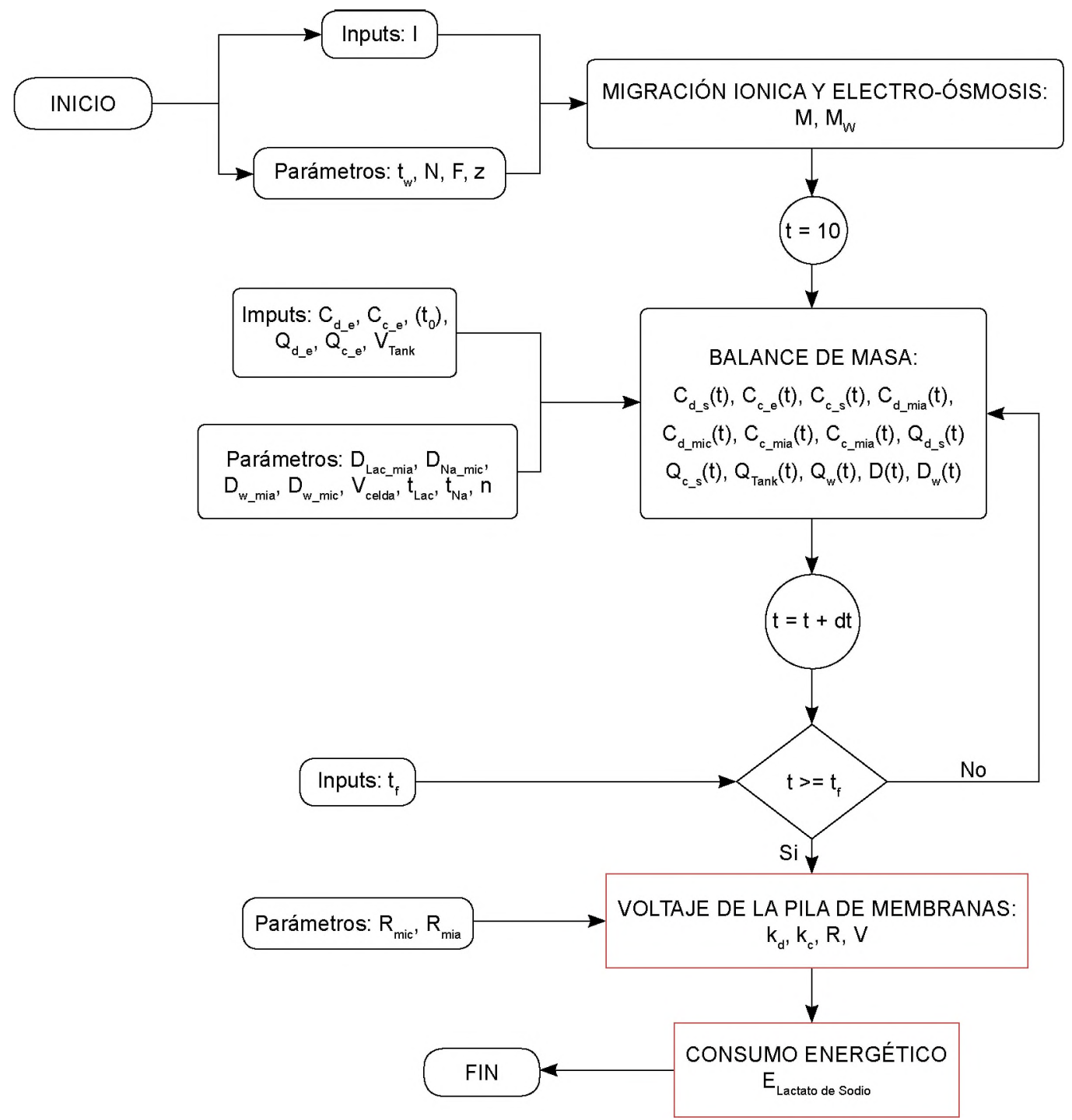

Figura 3. Algoritmo iterativo para solucionar todas las ecuaciones del sistema, como las ecuaciones que representan los fenómenos de masa, los balances de masa o el consumo energético del proceso.

Fuente: tomado de Bonet (2010).

\section{REFERENCIAS}

Bonet, N. (2010). Modelización de la concentración de salmueras de desalinización mediante procesos de electrodiálisis (tesis de pregrado). Ingeniería Química, Universidad politécnica de Cataluña, España.

Fidaleo, M., y Moresi, M. (2004). Modelling the electrodialytic recovery of sodium lactate. Biotechnology applied biochemistry, 40(2), 123-131.

Hábová, V., Melzoch, K., Rychtera, M., y Sekavová, B. (2004). Electrodialysis as a useful technique for lactic acid separation from a model solution and a fermentation broth. Desalination, 162, 361-372. 
Matsuura, T. (1993). Synthetic membranes and membrane separation processes. EE. UU.: CRC Press.

Mayorga, M., y Ariza, C. (2013). Diseño básico de una celda electrolítica para la defluorización de aguas residuales industriales por electrodialisis. Ingenium, 14(28), 41-59.

Medina, I. (2007). Tratamiento de aguas de producción con electrodiálisis (trabajo de grado). Escuela de Ingeniería Química, Facultad de Ingeniería, Universidad de los Andes, Mérida, Venezuela.

Ortiz, J., Sotoca J., Expósito, E., Gallud, F., García-García V., Montiel, V., y Aldaz, A. (2005). Brackish water desalination by electrodialysis: batch recirculation operation modeling. Journal of Membrane Science, 252(1-2), 65-75.

Thang, V., Koschuh, W., Kulbe, K., y Novalin, S. (2005). Detailed investigation of an electrodialytic process during the separation of lactic acid from a complex mixture. Journal of membrane science, 249(1), 173-182. 